\title{
Microbes in space
}

There's a lot of bacteria to consider in the microbiome - the Human Microbiome Project calculated that the normal human microbiome contains over 10,000 different species-and knowing who they all are can help researchers understand the interactions between microbes and their hosts, in sickness and in health. But "who's there" is just one question to ask when interrogating microbiomes. Jessica Mark Welch, a scientist at the Marine Biological Laboratory (MBL) in Woods Hole, MA and Gary Borisy at the Forsyth Institute in Cambridge, MA, are interested in identifying where all those bacteria are as well.

"Bacteria respond to their environment, and a key part of their environment is the other bacteria that are in there with them, the other bacteria that they're next to," Mark Welch explains. "We think that structure and function are very tightly related and that in order to really understand the function of microbial communities, we are going to have to understand their structure."

Much of the work on the spatial structure of bacteria in (and on) their hosts has occurred in the realm of theoretical biology. Different models attempt to predict how spatial organization helps microbial communities remain stable (or, not) over time and how that influences things like host-microbe coevolution and the impacts of the microbiome on health and disease. Mark Welch and her colleagues are putting that theory into practice. "We think that it's critical to actually look, to get experimental data to validate or test these various hypotheses," she says.

A new project was born a few years ago at MBL. "We set out to try to visualize basically all of the key microbes in a community simultaneously," she says. Their technique of choice-fluorescence in situ hybridization, or FISH - wasn't new to microbiology. Researchers have used colored fluorescent probes, or fluorophores, targeted to specific DNA sequences on bacterial chromosomes to identify microbes for decades, but the band-pass filters that were commonly used on microscopes could only tease apart a few microbes at a time. Researchers could glimpse how simple communities were assembled when only three or four types of bacteria were present, but natural systems tend to be much more complex.

After several years of development and testing, Mark Welch and her colleagues, including Borisy, the former director of the MBL, and Alex Valm, published a new imaging method that could distinguish up to 28 different fluorescently tagged microbes, which they dubbed Combinatorial Labeling and Spectral Imaging FISH, or CLASI-FISH (Proc. Natl. Acad. Sci. USA 108, 4154-4157; 2011). Unlike band-pass filters that typically only distinguish fluorescence between red, green, and blue wavelengths, spectral imaging can detect color across a continuous emission spectrum for each pixel in an image. That means more fluorophore options to tag different bacteria.

The proof-of-concept included a spatial analysis of microbes found in samples human dental plaque; a new paper published last October, in collaboration with Jeffrey Gordon and Nathan McNulty at Washington University in St Louis, MO, takes CLASIFISH to gnotobiotic mice (Proc. Natl. Acad. Sci. USA 114, E9105-E9114; 2017). The gnotobiotic mice contained just 15 known bacteria, to which the team tailored their fluorophores. After allowing 14 days for the bacteria to establish themselves in the mice, the team dissected cross-sections of the proximal colon (an area with high bacterial density), fixed the samples in formaldehyde and embedded them in methacrylate, and applied the FISH probes to image where each bacterium was in the sample.

Given the distinct spatial structures they observed previously in human dental plaque, they expected to see similar microbial divisions between the lumen - the tunnel space inside the colon-and the mucosa that lines it. But they were surprised to see that the bacteria were much more randomly mixed, though some associations did appear in small microhabitats. After looking back into the literature, "we realized that there are reasons to think that the colon would be a much more mixed habitat than we'd

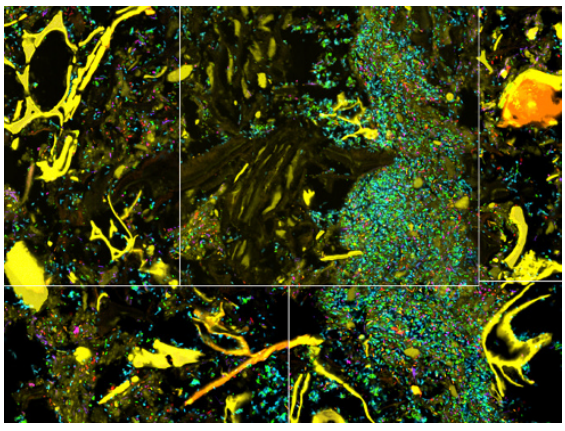

The colorful spatial organization of different microbes from the colonic lumen of a gnotobiotic mouse. Adapted with permission, Proc. Natl. Acad. Sci. USA 114, E9105-E9114; 2017.

expected," Mark Welch says. "The gut is a dynamic environment," she explains, with mucus and other cells constantly sloughed into the lumen where they are mixed with the contents of the gut that are being pushed through. "We think the spatial organization we see is a reflection of that."

With the technique to map microbial communities from living organisms established, Mark Welch would next like to look more at why the microbes end up where they do, by modifying factors like diet and the immune system in gnotobiotic animals to "see if we can really begin to identify the factors that influence spatial organization in the microbiota."

There's also still a lot more of the gut to look at, and in even more natural systems. Though gnotobiotic mice offer more complexity than an assemblage in a petri dish, they're still an intermediate step to the whole microbiome found in an organism. But as DNA sequencing becomes ever more inexpensive, Mark Welch expects their technique will be applicable with any animal they might want to work with in the future. "We could sequence the microbiota that's present in that particular animal and then develop and design probes specific for the bacteria that we find there in abundance."

Time to get to know the neighbors in the microbial neighborhood.

Ellen P. Neff 